\title{
CULTURAS DE CONSUMO DE ALCOHOL Y COCAÍNA: PRÁCTICAS Y SENTIDOS DE LA EXPERIENCIA FEMENINA JUVENIL METROPOLITANA
}

\author{
SEBASTIÁN BRAVO ViVEROS*
}

\begin{abstract}
RESUMEN
El texto presenta y analiza las distintas prácticas, dinámicas y sentidos que comprende el consumo festivo y recreativo de alcohol y cocaína para las mujeres jóvenes de la Región Metropolitana. Para aquello, se delinean y caracterizan las lógicas de uso de cuatro culturas de consumo (punk/hardcore, underground, alternativa y house/electrónica) en distintos territorios de despliegue y en diferentes momentos de las cadenas rituales de consumo. A su vez, rastreamos los distintos procesos de transgresión, flexibilización y continuidad de la normatividad social en el campo de las drogas, poniendo en relieve las tensiones que suscita el consumo femenino dentro de las culturas y sociabilidades juveniles. En ese sentido, sostenemos — a modo de hipótesis - que asistimos a la reconfiguración de las normatividades de género, anclada a un proceso de transformación de los patrones de consumo de drogas transnacionales, en el marco de la profundización del paradigma punitivo «contra las drogas» y de la gestión gubernamental de las juventudes.
\end{abstract}

PALABRAS CLAVE: CULTURAS DE CONSUMO, DROGAS, MUJERES JÓVENES

\section{CULTURAS DE CONSUMO DE ÁLCOOL E COCAÍNA: PRÁTICAS E SENTIDOS DA EXPERIÊNCIA FEMININA JUVENIL METROPOLITANA}

\section{RESUMO}

O texto apresenta e analisa as diferentes práticas, dinâmicas e sentidos que compreende o consumo festivo e recreativo de álcool e cocaína para as mulheres jovens da Região Metropolitana. Para isso, são delineadas e caracterizadas as lógicas de uso de quatro culturas de consumo (punk/hardcore, underground, alternativa e house/eletrônica) em diferentes territórios de ação e em diferentes momentos das cadeias rituais de consumo. Ao mesmo tempo, identificamos os diferentes processos de transgressão, flexibilização e continuidade da normatividade social no campo das drogas, destacando as tensões que provoca o consumo feminino nas culturas e sociabilidades juvenis. Nesse sentido, sustentamos - como hipótese que presenciamos a reconfiguração das normatividades de gênero fixada a um processo de transformação dos padrões de consumo de drogas transnacionais no contexto do aprofundamento do paradigma punitivo “contra as drogas” e da gestão governamental das juventudes.

PALAVRAS-CHAVE: CULTURAS DE CONSUMO, DROGAS, MULHERES JOVENS 


\title{
ALCOHOL AND COCAINE CONSUMPTION CULTURES: PRACTICES AND SENSES OF METROPOLITAN YOUNG FEMALES' EXPERIENCE
}

\begin{abstract}
The text introduces and analyses the different practices, dynamics and senses included in the recreational and festive alcohol and cocaine consumption for young women from Metropolitan Region, by means of drafting and characterizing their usage according to four different consumption cultures (punk/hardcore, underground, alternative and house/electronic), in different display territories of action and moments of ritual consumption chains. In addition, we traced the different transgression, flexibilization and continuity processes of social normativity regarding drugs, emphasizing the tensions raised by female consumption within the youth cultures and sociabilities. We state, therefore - as a hypothesis - that we are witnessing the reconfiguration of gender normativities, which are linked to a process of transformation of the consumption patterns of transnational drugs, in the context of the deepening of the anti-drugs punitive paradigm and governmental management of youths.
\end{abstract}

KEYWORDS: CONSUMPTION CULTURES, DRUGS, YOUNG WOMEN

«Donde manda capitán, muere, muere, muere, muere marinero, todos saben bien que es por dinero.

Me hago un asado, de faso, tomo sodeado, me paso.

Termino borracha, tengo buena suerte y mala racha».

(Asado de Fa, Sara Hebe)

\section{INTRODUCCIÓN}

El uso de sustancias psicotrópicas ${ }^{1}$ ha sido una práctica atávica de la humanidad. No obstante, es a partir de finales del siglo XIX y principios del siglo XX que se inicia el proceso

\footnotetext{
* Antropólogo Social, Universidad de Chile. Miembro del Núcleo en Acción e Investigación en Juventudes, Universidad de Chile. E-mail: sebast.bravo@gmail.com

${ }^{1}$ Usamos sustancias psicotrópicas como sinónimo amplio de sustancias psicoactivas.
} 
de masificación y expansión general del consumo de drogas entre las capas medias y populares en Occidente, fenómeno que determinó que ciertas sustancias psicoactivas dejaran de ser consideradas de uso exclusivo de las élites y las clases «minoritarias», y pasaran a ser de uso general (Luna-Fabritius, 2015). En efecto, este proceso estuvo vinculado al proceso de reorganización capitalista global en el período de posguerra y se tradujo en intensos procesos de migración que condicionaron la formación de nuevos actores, la reorganización de la estructura del trabajo, la crisis de los roles femeninos del modelo fordista y la aparición de emergentes condiciones de vida y relaciones urbanas que decantaron finalmente en la aparición de nuevos patrones de consumo.

En el caso particular de Chile, los procesos de visibilización del consumo femenino juvenil de drogas y la incorporación de mujeres en espacios de festivos y de ocio - fuertemente masculinizados - fueron fenómenos que se dieron paulatinamente hacia mediados del siglo $\mathrm{XX}$, los que estuvieron anclados a la influencia de la industria musical internacional, del rock \& roll, el movimiento de la Nueva $\mathrm{Ola}^{2}$ y los clubs de fans asociados a esta escena artística, además de los malones y peñas, donde era recurrente el consumo de bebidas alcohólicas, como el pisco o la grapa, y de marihuana y tabaco (cigarrillos).

Posteriormente, en la década de los 60 y 70, se dio una nueva transformación en el patrón de consumo juvenil. El uso terapéutico dio paso al comienzo de los usos lúdicos y recreativos intensivos, a través de la heroína en Europa y Norteamérica, además de la élite latinoamericana, alternando en las décadas siguientes las tendencias de consumo recreativo de otras drogas ya ilícitas, como la cocaína, las drogas de síntesis y la marihuana, en el contexto del nacimiento del movimiento hippie, el auge de los movimientos estudiantiles en la década del 60 en América Latina, los procesos revolucionarios y de descolonización en los países subalternos y las múltiples expresiones de la contracultura juvenil.

\section{a) Consumo femenino juvenil de drogas}

Las transformaciones estructurales de los patrones de consumo femenino y juvenil de las últimas décadas se han traducido en el incremento y visibilización del consumo femenino, el cual se ha configurado como uno de los procesos clave del escenario actual en el campo de las drogas. En particular, el consumo femenino y juvenil mundial ha estado marcado por una paulatina alza de la ingesta de alcohol y tabaco, una acentuada feminización del consumo de psicofármacos en el campo de las drogas lícitas y el sostenido crecimiento del consumo de cannabis, cocaína y nuevas sustancias psicotrópicas (NSP) en el campo de las drogas ilícitas.

Por otra parte, y en lo que respecta al actual panorama de consumo latinoamericano, encontramos grandes transformaciones producto de la era digital, la cual ha reconfigurado el estilo de vida y visión del mundo juvenil, esbozando rupturas generacionales y produciendo nuevas formas de socialización que impactan en la emergencia de nuevos patrones de consumo de drogas mediados en sus usos y modelos culturales por la virtualidad (Tapscott, en Feixa, 2006). Dentro de estos cambios, destaca la aparición y extensión de nuevas sustancias (Gómez, 2012), lo que ha repercutido en derribar el mito del consumidor de una sola sustancia (Calafat,

\footnotetext{
${ }^{2}$ Nueva Ola es el término con el que se denominaba en Chile al grupo de músicos e intérpretes que, durante los años 60 y hasta principios de los años 80, adoptaron la influencia musical del rock \& roll de Estados Unidos y la cultura pop de Europa.
} 
en Gómez, 2012), considerando el policonsumo como el nuevo patrón que establecen las y los jóvenes en relación con las drogas. Dentro de aquellas modalidades de policonsumo, el binge drinking (consumo en atracón) (Gómez, 2012) se ha convertido en uno de los fenómenos claves del consumo juvenil, en tanto el alcohol es la sustancia más consumida y, a su vez, la más elegida para comenzar la noche, y a la cual se le van sumando la ingesta de tabaco, marihuana, cocaína o drogas sintéticas. Sumado a lo anterior, ha aparecido una amplia gama de nuevas sustancias psicoactivas (NSP), que a menudo tienen propiedades químicas y/o farmacológicas similares a las sustancias controladas internacionalmente (CICAD, 2015).

Con respecto a los contextos en que se da el policonsumo, los jóvenes tienden a vincular el consumo de drogas con instancias de ocio y tiempo libre asociados a la diversión y reunión con los amigos, fundamentalmente en espacios privados. En tal sentido, y tal como señalan Ralet y el Grupo Igia (2000), el nuevo escenario de consumo juvenil se configura a partir de la generalización y masificación de determinadas pautas de ocio juvenil: la concentración en el fin de semana, el predominio de la noche sobre el día, su asociación a determinados lugares, etc., anclados en la creación de diversas culturas juveniles o los cambios en las anteriores, relacionadas con la diversión: predominio de determinadas músicas, estilos y estéticas, valoración de ciertos estados de ánimo, etc. (p. 126).

En lo que respecta a Chile, estas pautas de sociabilidad juvenil se dan en espacios que suelen construirse en torno a momentos de tranquilidad o euforia y energía, entendidos como aquellos momentos en que el carrete se da en casa y es más piola y tranquilo, u otros donde los carretes son prendidos/intensos, con harta gente (SENDA, 2012). En menor medida, se ha observado también que los espacios públicos son preferidos por aquellos jóvenes que consumen drogas, quienes declaran hacerlo «en la plaza/calle con amigos».

A su vez, el consumo de alcohol y marihuana tiende a aparecer actualmente como algo cotidiano dentro del contexto universitario, lugar donde ha pasado a convertirse en una práctica naturalizada, siendo parte de la socialización que experimentan los estudiantes dentro de ese espacio. No obstante, no sucede del mismo modo con sustancias como la cocaína o la pasta base, las cuales son rechazadas fundamentalmente por asociarse a niveles de adicción mayor. Dentro de aquello, además existen situaciones sociales preferentes para el policonsumo. Entre ellos, la previa, entendida como juntarse en algún lugar o sitio y allí ingerir cualquier tipo de bebida alcohólica mientras se hace tiempo, para ir o no, dependiendo del estado de alcoholemia, al boliche bailable. Se trata de una práctica común para las y los jóvenes de Chile y Argentina (Del Zotto et al., 2010). En particular, el carrete emerge como una de las prácticas más representativas y extendidas en la juventud chilena postdictadura, siendo un ámbito de sociabilidad transversal a los diferentes estilos y culturas juveniles (Matus, 2005).

b) Consumo sexuado: normalización, desviación y triple estigma de las mujeres consumidoras de drogas

En el caso particular de Chile, vemos la consolidación del rango juvenil como el mayor consumidor de drogas en nuestro país (SENDA, 2012, 2015, 2016). A su vez, en el caso del consumo de la Región Metropolitana, la zona urbana muestra que la diferencia entre el femenino y el masculino tiende a ser mayor, hecho que se expresa en que el consumo femenino de drogas ilícitas (marihuana y cocaína) es la mitad en comparación de los hombres. (SENDA, 2012, 2015). Aquello coincide con las tendencias internacionales que muestran que el consumo 
femenino está concentrado en el uso de drogas lícitas, a diferencia del masculino, enfocado las sustancias ilícitas (Romo y Pérez, 2013). En ese sentido, casos como el alcohol y la cocaína son ejemplos paradigmáticos de drogas donde el crecimiento de su consumo constituye una equivalencia en las prevalencias históricas entre sexos, hecho que da luces en torno a la existencia de construcciones de género, sociales y generacionales que siguen operando en la práctica.

Sumado a lo anterior, es posible rastrear la existencia de un triple estigma femenino asociado al consumo de drogas. De este modo, las mujeres consumidoras han quedado circunscritas a la imagen de la «mujer adicta», la cual aglutina un triunvirato de imágenes sociales como la mala madre (Romo, 2005), la delincuente y la prostituta, categorías que terminan por revelar la estrecha articulación que existe entre prácticas de consumo, roles de género y sujetos fuera de la norma, y cuyos fundamentos se sustentan sobre la base de imágenes estereotípicas en torno a la prostitución y la desviación de las feminidades hegemónicas y socialmente aceptables (Rosenbaum y Murphy, 1990), teniendo como efecto la construcción de un imaginario social negativo de las mujeres consumidoras y un marcado rechazo social frente a sus prácticas de consumo.

Esta misma apreciación vale para el consumo y dependencia de sustancias lícitas (el alcohol o el tabaco principalmente), ante la cual la mujer se halla constreñida por una estrechez superior de los márgenes de la norma masculina. Así, la desviación respecto a aquella normatividad se relaciona enseguida con sus roles sociales de madre, ama de casa, en conjunto con sus expectativas, sus responsabilidades familiares, etc. (Rekalde y Vilches, 2003). En ese sentido, estos procesos marcan y refuerzan la articulación de un imaginario social entre sustancias psicotrópicas, toxicomanías y delincuentes o agentes antisociales dentro de la sociedad chilena (Fernández Labbé, 2011), fundamento histórico que ha servido para la construcción de las consumidoras de drogas como enemigas internas del Estado y en cierto sentido, desestabilizadoras del orden familiar y político. Ejemplo directo de aquello es el creciente aumento de mujeres presas en América Latina, en un proceso de feminización de los encarcelamientos de los delitos asociados a drogas (Markez et al., 2011).

En consecuencia, y dado lo anterior, la construcción del ser mujer en nuestra sociedad influye en la forma en que ellas se aproximan y consumen los distintos tipos de drogas, y los espacios que construyen para ello. No obstante, y a pesar de las múltiples hipótesis (McDonald, 1994; Babcock, 1996; García-Mina y Carrasco, 2007; Wilsnack et al., 2000; Peréz y Correa, 2011) que han intentado explicar las tendencias divergentes en el uso de drogas dentro de la literatura, todavía persiste un sesgo androcéntrico y adultocéntrico específicamente en las principales investigaciones relacionadas con drogas (Garber y McRobbie, en Feixa, 2006; Ettore, 1992; Alpízar y Bernal, 2003), lo cual configura un escenario de invisibilización de los motivos, deseos y significados que estructuran el uso de las jóvenes consumidoras. En ese sentido, el presente texto intenta indagar en el discurso de las drogas (Ibáñez, 1991), en un intento por reconocer el otro discurso, donde se rescata la propia experiencia del sujeto desde dentro (Ghiardo, 2003). Es a partir de aquello que abordamos las prácticas, dinámicas y sentidos que guían y dan forma a las experiencias de mujeres jóvenes en la Región Metropolitana.

\section{ENFOQUE TEÓRICO-METODOLÓGICO}


Abordaremos las culturas de consumo de drogas ${ }^{3}$ como el entramado de relaciones sociales, de género, generacional, de clase y territorial que comprenden la serie de modos de hacer en torno a la droga, y que engloban a todas las acciones y actividades asociadas a la adquisición, preparación, ingesta y actividades de postconsumo de las distintas sustancias. A partir de aquello, puede comprenderse que el proceso de consumo de alcohol y cocaína es una cadena o secuencia de consumo a partir del cual se elaboran redes de significación en torno a él, las cuales configuran saberes, sistema de valores, roles y patrones de acción histórica y contextualmente construidas, y que sirven como marco de sentido para las propias jóvenes mujeres consumidoras dentro del contexto juvenil contemporáneo.

Por otra parte, las culturas de consumo aparecen también como campos simbólicos en conflicto, en los que, desde diferentes posiciones, se definen y redefinen de manera constante y antagónica los sentidos y las interpretaciones. Dentro de aquello, diferenciamos prácticas como las operaciones diarias de las consumidoras, es decir, los «modos de hacer y de utilizar» las sustancias descritas. De manera más específica, las prácticas se definen como esquemas de operaciones, de manipulaciones técnicas y actos que apuntan al uso, apropiación y práctica de todo objeto producido y que se caracterizan por constituirse como maneras de hacer fragmentarias, insinuadas, escondidas y muchas veces dispersas, y que no necesariamente responden a marcos de comprensión institucionales y/o ideológicos (De Certau, 1996). A partir de aquello, los significados construidos operan como nodos de sentido dentro de un campo en constantes dinámicas de transformación, hibridación y conflictividad, donde se modelan distintas interpretaciones de las prácticas de consumo y su inteligibilidad.

Sumado a lo anterior, entendemos la juventud como una categoría política estructurada a partir de un entramado de relaciones de poder, que constituyen la práctica de consumo de alcohol y cocaína de las mujeres jóvenes. En consecuencia, esta construcción sociohistórica, en primer término, debe entenderse como un concepto situado y en donde cada sociedad define a la «juventud» a partir de sus propios parámetros culturales, sociales, políticos y económicos, por lo que no hay una definición única (Alpízar y Bernal, 2003). Es por eso que debe hablarse de juventudes, como categoría múltiple y en donde las jóvenes no constituyen un grupo homogéneo, totalizado y estabilizado, sino más bien una construcción (producción) relacional, en constante transformación y modulada por los distintos sistemas de clasificación y estructuras de poder. En el caso de Chile, las culturas de consumo son entidades atravesadas fuertemente

\footnotetext{
${ }^{3}$ Ahondar en el fenómeno de las drogas es reconocer un entramado complejo de discursos, prácticas y actores en tensión. No obstante, una definición expresiva de la complejidad del fenómeno entiende las drogas como aquellas sustancias químicas que se incorporan al organismo humano, con capacidad para modificar varias funciones de este (percepción, conducta, motricidad, etc.), pero cuyos efectos, consecuencias y funciones están condicionados, sobre todo, por las definiciones sociales, económicas y culturales que generan los conjuntos sociales que las utilizan (Romaní, 1999, p. 53). En ese sentido, es crucial además entender que las consecuencias, funciones, efectos y significados de estas sustancias son el producto de las definiciones sociales, culturales, económicas y políticas que las diferentes formaciones sociales (grupos, colectivos e individuos) elaboran, negocian y/o disputan en el marco histórico en el que se sitúan sus prácticas (Sepúlveda, 2011). En ese marco, enfatizamos un abordaje sociosimbólico, el cual entiende a las drogas como mercancías que operan como marcadores simbólicos o depositarios de significados (Valenzuela et al., 2009), a la vez que se comprenden como parte, la práctica de consumo vinculada al estatus y la estructura social de las consumidoras. En otras palabras, las drogas se consideran desde los significados que simbolizan para las consumidoras, como desde las relaciones sociales que sustentan entre jóvenes.
} 
por una matriz adultocéntrica y patriarcal (Duarte, 2000), en donde las diferencias y asimetrías que se dan en el consumo de drogas entre hombres y mujeres están vinculadas a un sistema de género (Rubin, 1986) y a un régimen de juvenilidades (Aguilera Ruiz, 2014) que, a través de distintos dispositivos de gobierno, produce un determinado sujeto como efecto de una clasificación y delimitación de la experiencia humana.

En cuanto al enfoque metodológico, este comprendió una estrategia cualitativa, combinando la técnica de entrevistas en profundidad y la etnografía multilocal (Marcus, 2001), en la indagación de las culturas de consumo y experiencia de consumo de alcohol y cocaína de diez mujeres jóvenes de la Región Metropolitana. ${ }^{4}$

\section{ADQUISICIÓN Y PROCESO DE CONSUMO}

El proceso de adquisición tanto de alcohol como de cocaína se estructura en torno a dos principios transversales que se anclan en determinaciones espacio-temporales.

El primero hace referencia al principio de proximidad, vinculado a la maximización del tiempo utilizado en adquirir una sustancia y que se traduce concretamente en la valoración de lugares de adquisición cercanos a las consumidoras o a los puntos de encuentro o de carrete juvenil. Aquello se sustenta en una lógica de aceleración social, que concibe el tiempo como un bien escaso y de rápido consumo, por tanto, se produce una búsqueda por «ahorrar tiempo» de compra, en el marco de los múltiples desplazamientos que algunos grupos juveniles realizan dentro de su circuito de carrete metropolitano y que los lleva a moverse de carrete en carrete. Por su parte, y en segundo lugar, el principio de portabilidad comprende los procesos de maximización del espacio, cuya consecuencia es la valoración y búsqueda de soportes físicos de drogas y sustancias (latas, cajas, botellas, bolsas, etc.) de tamaño reducido, fácilmente desagregables, de fácil consumo y de rápido resguardo ante posibles sanciones legales o traslados del grupo de un lugar a otro. Cabe comprender, por tanto, que los principios de proximidad y portabilidad operan como claves tácticas, vinculadas a la movilidad y temporalidad juveniles que estructuran las distintas fases del consumo al interior de las culturas de consumo y que, articulados y mediados por otros factores, constituyen premisas básicas para los modos de consumo de las jóvenes.

Sumado a lo anterior, también pudimos identificar la existencia de dos circuitos de circulación mercantil que operan en las culturas de consumo y que están estructuradas en torno al estatus legal de las sustancias que se consumen. El primer circuito, reconocido como oficial, es el comercio lícito, el cual comprende la venta de las sustancias legalizadas, como alcohol o tabaco, y que abarca puntos multicéntricos de adquisición y consumo. Este circuito sustenta su

\footnotetext{
${ }^{4}$ Para resolver el obstáculo referente a la investigación de poblaciones ocultas, hemos utilizado procedimientos de muestreo intensivos que se basan en diseños de cadenas de referencia (Spreen, 1992) o bola de nieve (Biernacki y Waldorf, 1981). No obstante, y dada la naturaleza de la presente investigación, además optamos por complementar la técnica de bola de nieve con la determinación de perfiles etnográficos bajo criterios tipológicos (Funes y Romaní, 1985; Spotts y Shontz, 1980). La determinación de los perfiles etnográficos se dio siguiendo el criterio de niveles socioeconómicos según tres segmentos simplificados: estrato alto, medio y bajo, y que profundizamos a través de la distinción de cuatro culturas de consumo asociadas a estilos juveniles (punk/hardcore, alternativa, house/electrónica y underground). En cuanto a la estrategia de muestreo, este se dio en torno a una estrategia de selección no representativa y guiada por principios de saturación, mientras que el análisis se delineó a partir del método de análisis de contenido.
} 
amplitud en el estatuto legal de las sustancias que hace circular y, por tanto, su naturaleza es siempre visible, pública, legítima y también naturalizada, donde opera una lógica de elección racional. Por otra parte, tenemos el subcircuito ilícito, que también forma parte del circuito comercial lícito, pero de manera oculta, como forma de evadir la sanción social y legal que acarrea cualquier transacción con sustancias ilícitas, como la cocaína, marihuana, etc. Este último circuito, como vemos, se basa en redes de confianza y resguardo que permitan desactivar los procesos de estigmatización y que opera en torno a una lógica desregulada y en donde las barreas de acceso son más potentes, de modo que el uso del capital social como forma de ampliar la elección de consumo pasa a ser un factor relevante. No obstante, ambos circuitos se basan y amplifican en dinámicas de configuración de estatus intragrupales y delimitaciones fronterizas intergrupales a partir del consumo.

En lo que respecta a las distintas prácticas de consumo en sí mismas, estas se articulan como cadenas de rituales de interacción (Collins e Iranzo, 2009), es decir, mecanismos que forjan una emoción y una atención conjuntas, generando una realidad temporalmente compartida y un estado de intersubjetividad intensificada, y a partir de las cuales se genera una realidad simbólica compartida autónoma del ambiente circundante, orientadas por códigos rituales, papeles y roles de acuerdo con un guion social determinado y que construyen un guión moral que viabiliza ciertas dinámicas al interior de los grupos juveniles y entre las distintas consumidoras, codificando así la manera en que se procesan colectivamente los efectos y la correcta forma de habitar el cuerpo y el territorio cuando se está borracha o jalá.

Dado lo anterior, es posible entender que las cadenas de rituales de consumo de drogas sustentan su eficacia simbólica en los fundamentos de comensalidad e identidad, como procesos de identificación/exclusión que van estructurando y organizando a la comunidad emocional y social que se crea a través de ellas. En efecto, a continuación, esquematizamos las principales etapas del proceso de consumo. 


\section{Prenderse}

Rondas iniciales de consumo de marihuana y bebidas alcohólicas de bajo grado alcohólico (generalmente cerveza). Se complementa con el consumo de cigarrillos o tabaco para rolar.

\section{Repeticiones}

Dependiendo de los efectos subjetivos e intersubjetivos del grupo, se pasa al consumo de bebidas alcohólicas de mayor grado alcohólico (vino o destilados, como pisco o vodka). Otras jóvenes oprtan por seguir consumiendo bebidas de bajo grado alcohólico. Se producen otras rondas de consumo de marihuana y también dinámicas de juego en el marco de binge drinking.

Muchas veces, el rito de consumo puede transformarse en una previa.

\section{Expansión social}

Los efectos combinados del alcohol, la marihuana y el tabaco llegan al cénit ritual, en donde las interacciones sociales se producen con mayor intensidad. Se flexibiliza el guion hegemónico y se esbozan otras dinámicas sociales y simbólicas.

Se continúa el consumo de bebidas alcohólicas de alto grado alcóholico (vodka, pisco, ron) y se puede complementar con el consumo de bebidas de muy alto grado alcohólico como consumo límite (tequilazos, cortos de destilados solos, etc.).

\section{Intensificación}

Dependiendo de los efectos del policonsumo de alcohol y marihuana y de las interacciones sociales derivadas, se produce el consumo de cocaína, que puede dosificarse en una ronda o en varias durante la noche.

Las interacciones sociaes se intensifican, llegando a un cénit de energía. Ciertas culturas de consumo mediatizan eso a través del baile; otras, a través de la conversación.

Asociado a lo anterior, se desencadenan cadenas seguidas de policonusmo de más alcohol, tabaco y marihauna.

\section{Cierre}

Posterior al consumo de cocaína y a la disminución del efecto, puede darse el cese del consumo, ya sea dejando de consumir cualquier sustancia o espaciando el consumo de alcohol en el tiempo. Generalmente, también las consumidoras retoman el consumo de bebidas alcohólicas de bajo grado alcohólico (cerveza), como forma de ir bajando el consumo paulatinamente en el contexto del after hour. En otros casos, se sigue bebiendo y consumiendo cocaína hasta llegar al punto de desmayarse (apagar tele) o quedarse dormida.

Fuente: esquema de elaboración propia, sobre la base de la experiencia etnográfica y entrevistas y semiestructuradas. Cabe destacar de antemano que esta esquematización se da como formulación formal y, por 
tanto, no debe comprenderse de manera lineal, sino con procesos simultáneos, yuxtapuestos y en constantes saltos de una fase a otra y viceversa.

\section{a) Guion hegemónico: siempre señorita, siempre femenina}

A lo largo de las distintas etapas de las cadenas de consumo, es posible rastrear la constitución de un guion que estructura las principales dinámicas y prácticas de consumo de las jóvenes. En ese sentido, nos interesa abordar el guion hegemónico que rige este proceso, en primer lugar, como un cuerpo normativo que encarna una serie de distinciones morales vinculadas a procesos de subjetivación (Foucault, 1990) y que se dan ancladas a moralidades de género (gendered moralities) (Tolvanen y Jylhä, 2005) y, en segundo lugar, como una herramienta cultural que, depositaria de una noción instrumental, les permite intervenir en el mundo

A continuación, repasamos tres ejes fundamentales de este guion hegemónico:

i) Maternización y persistencia del rol de cuidados

Dentro del proceso de investigación, no participaron madres consumidoras, no obstante, es posible identificar una lectura mayoritariamente negativa de parte de las jóvenes consumidoras de la relación entre maternidad y consumo de alcohol y/o cocaína. Esta lectura sigue un arco argumentativo desde el rol pedagógico de la maternidad, hasta el fundamento científico en torno al riesgo de las hijas e hijos, no obstante, y a pesar de la diversidad de campos de los que se extraen los argumentos, este tipo de discursos sostiene su fuerza argumentativa en una noción moral de la conducta e identidad femenina, que se traduce en la imposibilidad de articular el rol maternal y de cuidados con del consumo de drogas de las mujeres.

No obstante, en una dimensión práctica y a pesar de lo anterior, las mujeres consumidoras en los contextos de carretes (ya sea mixto o en contextos de homosocialización) siguen ejerciendo un rol de cuidados en los grupos juveniles. Las mujeres previenen a sus pares de los riesgos de la falta de control y regulación del consumo de alcohol por, sobre todo:

Sí, hueona (sic), tenís que comer algo o si no te vai’ curar, pero raja. Yo te voy a obligar. (Amiga de C. a C. Etnografía Pircas FAU. Universidad de Chile).

En el escenario de los distintos espacios de consumo y especialmente aquellos privados de consumo, las mujeres asumen la tarea de cuidar a las y los otros consumidores, ya sea advirtiéndoles de ciertos riesgos derivados del consumo u ayudándolos en caso de que tengan efectos no esperados, como vomitar, caerse, etc.

ii) Modelos de belleza y gestión corporal femenina

Ligado a lo anterior, el proceso de maternización y persistencia del rol de cuidados como parte de lo femenino se construye en relación con la gestión del cuerpo femenino y la valoración de un modelo de belleza determinado. Ejemplo de ello es que, en el caso del consumo de cocaína, el guion hegemónico opera como estigma estético, a partir del cual se lleva a jerarquizar la belleza según criterios de moralidad. De este modo, y tal como plantea Vigarello (2005), puede verse cómo en su genealogía, la concepción de belleza se encuentra atravesada 
por relaciones de poder que son las que van determinando su valor: lo bello/bueno y, por oposición y límite, lo feo/malo.

Es que igual la coca es fea. (P., 19 años. Estudiante de Ingeniería en Alimentos/barista Starbucks. Pudahuel).

En este punto, cabe destacar que el jalar coca tensiona directamente la representación del modelo de belleza basado en la imposición de estándares y gestos corporales precisos como inherentemente femeninos.

Tal como señala Muñiz (2014), la belleza femenina en Occidente es la representación de virtudes morales o espirituales, en este sentido, la belleza asociada a la bondad es una condición de la feminidad y, por tanto, se convierte en una obligación moral para las mujeres. En ese sentido, es necesario ser bella para ser buena, ergo, es necesario ser buena para ser mujer.

El modelo de belleza, por tanto, actúa como dispositivo de normalización y subjetivación en el campo de las drogas que opera como norma estética y moral en el comportamiento de las mujeres consumidoras.

Otra dimensión de este vínculo es la relación entre consumo de alcohol y la delgadez. El alcohol, sobre todo en estratos altos, es visto como un enemigo del cuerpo perfecto y del modelo de belleza hegemónico, caracterizado por un cuerpo juvenilizado, delgado y flexible. En ese sentido, la sociedad prepara y alienta a los individuos para procurarse un cuerpo que ostente juventud, delgadez y sensualidad; en tanto que debemos rechazar el cuerpo decadente, envejecido o discapacitado (Muñiz, 2014; Le Breton, 2002).

Aparte de irme a acostar y sentir todo el copete adentro mío, además que me baja la hueá (sic) de que estoy gorda. Siento que uno puede permitírselo si se lo va a quemar. (H., 28 años. Guionista de TV y cine. Providencia).

El rechazo de la gordura y la valorización de la delgadez como nodo central del estigma estético asociado al consumo de alcohol plantean otra arista de la performatividad de género asociada a las culturas de consumo. En tal sentido, la delgadez obligatoria se constituye como un signo y reforzamiento de la femineidad y del ser buena mujer.

Este canon, asociado a la cultura electrónica/house y a la cultura alternativa de estratos medios y altos en general, opera sobre la base del modelo de belleza occidental y cuyo ideal está dado por un cuerpo perfecto de piel blanca, delgada y alta. Precisamente, esta representación de la mujer excluye simbólicamente a una amplia y heterogénea gama de cuerpos que no cumplen con esta norma. A partir de eso, varias consumidoras limitan su consumo como forma de no engordar y mantener una imagen corporal acorde al canon estético.

iii) Lo femenino como subalternidad simbólica 
Otra característica que complementa el guion hegemónico apunta a vincular lo femenino con la dulzura y la suavidad. Aquel discurso se basa en que las cadenas de rituales de policonsumo se dan siguiendo una cierta lógica de progresión, que parte del consumo de sustancias más suaves — ya sea marihuana o alcohol — para ir escalando a otras bebidas de mayor grado alcohólico u otra droga, como la cocaína u otras, según una lógica que considera al jale como una droga con un efecto más rápido y más fuerte que las anteriores. Aquella práctica de policonsumo opera a través de la representación masculinizada del consumo de drogas, en la cual las mujeres deben partir con sustancias suaves y paulatinamente ir ascendiendo a sustancias más fuertes. Bajo este argumento, las mujeres deberían seguir una norma moral de recato durante el consumo simbolizada por las drogas suaves, el cual que debería irse flexibilizándose paulatinamente, ascendiendo hasta llegar al quiebre del guion hegemónico dado por los efectos de las drogas más duras como la cocaína. Aquello supone que la experiencia de las drogas duras está reservada solo para los hombres como actores que poseen fuerza y aguante para resistir sus efectos.

Sumado a lo anterior, los denominados tragos de minas ${ }^{5}$ constituyen otro eslabón del binarismo de género femenino/masculino. Bajo ese argumento, este tipo de bebidas alcohólicas se posiciona en el polo inferiorizado de esta dicotomía y, por tanto, en vínculo directo con la feminidad asociado a los atributos de dulce y suave. En contraposición, los tragos para hombres se posicionan en el polo superior y se caracterizan por ser de mayor grado alcohólico, con un sabor más amargo, es decir, más fuerte y también más bueno. Esto reafirma una noción moral de comportamiento que deriva en la identificación de las cualidades de la mercancía consumida con los sujetos sociales consumidores, es decir, la vinculación de ciertos atributos sígnicos de ciertas bebidas alcohólicas, como la dulzura o la suavidad, con la construcción simbólica y moral denominado mujer: las mujeres, por tanto, deben ser suaves como los tragos que consumen. En consecuencia, lo que se juega en este ritual de progresión es la división sexual del imaginario de las drogas, en la cual la femineidad se construye desde la carencia de experiencia y de fuerza ante la masculinidad como canon de consumo.

En definitiva, la existencia del guion hegemónico asociado al consumo se traduce en una constelación de valores que determinan y legitiman cómo debe ser una «mujer». En ese sentido, tanto el valor de la abstinencia como el de la maternidad, la belleza, el recato, la dulzura o la pasividad se apoyan en una noción biopolítica del cuerpo femenino como empresa personal, enmarcado en un proyecto individual y más aún en un valor en sí mismo para la sociedad. En ese sentido, el consumo de drogas de las jóvenes adquiere en el guion hegemónico la dimensión de una dicotomía entre un cuerpo incorruptible y un cuerpo corrupto, ultrajado por el consumo de alcohol y sobre todo de cocaína. Es por esto que la preservación de la integridad social encarnada en el cuerpo incorruptible femenino supone directamente la preservación del estatus ontológico del sujeto para las jóvenes. En tal sentido, la transgresión de ciertos pilares de aquel guion termina por desestabilizar esa categoría, convirtiendo a la consumidora en una mala mujer o en una no-mujer.

\section{TERRITORIOS PSICOTRÓPICOS Y SIGNIFICADOS SOMATO-POLÍTICOS}

\footnotetext{
${ }^{5}$ Coloquialmente, se denominan tragos de minas o tragos de mujeres a las bebidas alcohólicas como el pisco sour, daiquiris, mojitos, entre otros, que se caracterizan por tener menos alcohol, de textura más ligera y con una base frutal o azucarada en la mayoría de los casos.
} 
Las culturas juveniles que se congregan en torno al carrete y el policonsumo de drogas poseen una dinámica en que las sustancias son compartidas colectivamente, en una cadena ritual de consumo que va constituyendo afinidad y legitimidad social entre las jóvenes integrantes del grupo. En esta investigación delineamos tres tipologías de espacios dentro del continuum de la sociabilidad juvenil: espacios públicos, espacios intersticiales y espacios privados, los cuales se construyen en relación con la potencia y capacidad de despliegue e impugnación que ejerce el guion hegemónico en el proceso de configuración de los territorios psicotrópicos en la ciudad.

A partir de lo anterior, esquematizamos una lógica de uso de cada espacio social asociado a la práctica de consumo que se viabiliza, a partir del cual pretendemos delinear algunos sentidos de consumo de las cuatro culturas de consumo relevadas en este texto (punk/hardcore, alternativa, underground y house/electrónica).

\section{a) Espacios públicos: potencia y movimiento}

Los espacios públicos apropiados y habitados por las jóvenes dentro de la Región Metropolitana se circunscriben a zonas donde predominan los discoteques/pubs/bares y lugares para «carretear», los que comprenden un circuito festivo central y circuitos festivos periféricos. ${ }^{6}$ Parte de los espacios públicos que las distintas culturas de consumo y mujeres ocupan y se apropian es la vía pública, tales como parques/plazas o la misma calle. Dentro de este escenario, destaca la presencia de grupos de jóvenes punk/hardcore otros asociados al vacile. Estas culturas de consumo cohabitan generalmente en el bandejón central de la Alameda, desde Los Héroes hasta Estación Central, además de habitar distintas plazas y parques del sector surponiente, como son el Parque O’Higgins, Plaza Brasil, San Miguel, Plaza Puente Alto, entre otros.

En específico, en el caso de la cultura punk/hardcore ${ }^{7}$, los efectos del alcohol se dan en función del compartir, en donde este último sienta su significado en la capacidad de desinhibición social que ejerce en el colectivo, a partir de lo cual se da la posibilidad de desviarse de la normativa social y, con eso, reforzar la identidad crítica y de transgresión con que se construye el movimiento punk. En tal sentido, el alcohol (preferentemente cerveza y vino tinto) opera como lubricante social, que aglutina a los diferentes miembros del grupo punki en torno a una dinámica móvil que inaugura escenarios de disrupción contingentes como es el

\footnotetext{
${ }^{6}$ El circuito festivo central de la Región Metropolitana comprende el barrio Bellavista, asociado al eje Pío Nono y calles aledañas; el barrio Brasil, Plaza Ñuñoa, barrio República, y en el sector Oriente, el barrio El Golf y el eje Providencia. En lo que respecta a los circuitos festivos periféricos, estos se constituyen en torno a ejes locales y lugares como discoteques/bares/pubs, en comunas como Quilicura, Maipú, La Florida, Peñalolén, entre otras.

${ }^{7}$ En Chile, el punk nace a partir de los 80, en un contexto dictatorial, y se origina como movimiento contracultural de resistencia, extendiéndose paulatinamente hasta consolidarse en el 2000 como un estilo reconocible públicamente, con una escena de tocatas permanente y anclado en un circuito de casas okupas y grupos conocidos y en constante renovación. En la última década, el punk ha virado hacia el hardcore, en un giro que intenta preservar la naturaleza crítica, fraterna y autónoma del punk original, cooptado por la mercantilización del estilo. Este nuevo estilo se caracteriza por una música ultrarrápida, de sonoridad dura y con letras de crítica directa contra el poder, que mezcla la estética del punk original con otras alternativas, skinhead o del heavy metal.
} 
vacile, que encarna dinámicas de creación ligadas al circo, teatro o diálogos de humor intenso asociados a discursos de crítica y transformación social.

En lo que respecta a espacios públicos e intersticiales, como casas okupa o centros sociales recuperados, una traducción relevante de la desinhibición etílica es el mosh, slam o pogo, baile caótico colectivo en donde se libera energía a través del movimiento de los cuerpos que caminan en círculos, entrechocándose, corriendo, etc. En efecto, se da un proceso de desestructuración del principio de individuación que rige los cuerpos diariamente para sublimarlos en una práctica colectiva donde estos se ponen en movimiento, en una marea que choca con otra, se balancea, esquiva y acelera, y que, a través de su encuentro, refuerza lazos y crea afinidades móviles y contingentes con otros punkis. No obstante, la construcción de los territorios psicotrópicos se da a partir de lógicas de género, en tanto la mayoría de los participantes en el mosh son hombres que siguen una dinámica de violencia legitimada, en la cual son frecuentes los puñetazos, patadas y empujones en torno al círculo caótico en que se transforman los participantes. Esto se traduce en que el alcohol y la potencia corporal y desinhibición social, simbolizada en el mosh, se construyen en torno a una dinámica sutil en donde a las mujeres, ya sea por considerarse menos fuertes que los hombres o como mecanismo de protección, se las excluye.

Con respecto a la cocaína, los punkis lo consumen como una sustancia de energización, que potencia el movimiento en el mosh, que, en conjunto con el alcohol, permite seguir en sintonía. En ese sentido, el jale no solo es consumido de manera instrumental, sino que reviste una intensificación de las experiencias de baile en tocatas o encuentros en espacios públicos.

b) Espacios intersticiales: entre la prolongación del goce y el «darlo todo» hasta morir (... y revivir)

Reconocemos espacios intersticiales como aquellos que se caracterizan por la coexistencia del guion hegemónico y de dinámicas de transgresión y flexibilización de esta norma, en un juego de ida y vuelta, a partir del cual se constituyen pequeñas zonas temporales o espaciales en donde no opera el guion hegemónico. En ese sentido, se da un proceso de convivencialidad de ambos guiones y la configuración de territorios psicotrópicos diferenciales en un mismo espacio en uso.

Espacios como los que describimos son la pista de baile de alguna discoteque, el living o un patio en un carrete de casa.

Dentro de estos espacios, las mujeres jóvenes comparten un uso diferencial de las distintas sustancias y, por tanto, construyen de distinta forma los territorios que habitan. La cultura de consumo electrónica/house, ${ }^{8}$ por ejemplo, ha tendido a acentuar el polo expresivo

\footnotetext{
${ }^{8}$ Las culturas de consumo asociadas a la escena electrónica/house surgen como efecto de los procesos de globalización y el desarrollo de la tecnología musical electrónica en Europa y Estados Unidos durante la década de los 80. La llegada a Chile de esta escena se da de la mano del desarrollo de las primeras fiestas ancladas a los circuitos centrales de diversión juvenil de la Región Metropolitana a comienzos de los 90, durante la época de la democracia tutelada, y que se dio a través de la experiencia cultural y musical de hijos/as de exiliados/as en Europa. En ese contexto, se da la aparición de los primeros clubes de música electrónica, en conjunto con la extensión de una incipiente industria musical electrónica que se desarrollará durante la década del 90 y que se verá constantemente alimentado por la proliferación de fiestas masivas y la venida de múltiples bandas y DJ de la escena internacional durante la década del 2000. Actualmente, la escena electrónica agrupa a diferentes grupos
} 
de las experiencias festivas de consumo, en donde tomarse una piscola se vincula directamente con la experiencia del baile asociada a la música.

Como de habitar sin tener que pensar en algo tan específico... lo asocio mucho a la música en verdá. (H., 28 años. Guionista de TV y cine. Providencia).

Por lo tanto, lo relevante del efecto del alcohol en esta dimensión remite a un ensamblaje, a una articulación entre una experiencia corporal de inmanencia y contingencia orgánica, que pone en sintonía los gestos y el movimiento perpetuo de los cuerpos jóvenes, con un tempo que reconstruye la relevancia simbólica y política de lo corporal como punto de gravedad, como anclaje de la experiencia que escapa de la experiencia moderna y cotidiana del futuro y que somete a las jóvenes al pálpito de un presente continuo, ampliado a través de las vibraciones sonoras.

Con respecto a la cocaína, el efecto psicotrópico se traduce en mayor energía, una recarga de ánimo que permite darle continuidad a la experiencia de baile. En tal sentido, el significado del jale como tecnología simbólica apunta a prolongar las capacidades del cuerpo, ampliando el umbral de movimiento y el tiempo de baile. Opera, en efecto, como la sustancia de la resistencia, pero no en torno al imaginario masculino del aguante, sino como sustancia que permite la prolongación del goce. Así, el significado de la cocaína dentro de esta cultura radica en la capacidad de atenuar la sensación de cansancio derivado, primero, de la rutina cotidiana y de la propia experiencia de baile que comprende esta cultura. En ese sentido, el jale es una sustancia vital para la mantención de la interacción social y de la apropiación festiva del espacio.

La traducción de la experiencia festiva, además, afinca su territorio primario de operación en el lenguaje corporal del baile, que desplaza lo oral para restituir la intraductibilidad del movimiento como forma de transgresión. Es precisamente en ese intersticio social que construye la música electrónica en la pista de baile como territorio psicotrópico, que aflora una simbiosis asociada al alcohol/cocaína/drogas de síntesis con los sonidos como flujos de ondas, que desfiguran los segmentos corporales como asistentes y que los remueven como partículas (Sepúlveda y Romaní, 2005). Se da, por tanto, una dinámica de contagio, de expansión rizomática del movimiento como suerte de infección que se amplifica como gesto repetitivo y que, en su diseminación colectiva, agudiza las sensaciones, convirtiendo todo en ritmo. El carrete, el consumo de drogas y la música se vuelven un pálpito indisoluble entre cuerpo y sujeto, que solo puede traducirse como cuerpo en movimiento.

Esta dinámica de contagio de cuerpos en torno a la cadencia de sintetizadores comprende una práctica alterna en tanto pone de manifiesto de nuevo la importancia de lo corporal como parte de la experiencia humana, oponiéndose a la invisibilidad del cuerpo y los rituales de borronamiento cotidiano (Le Breton, 2002), que promueven la distancia corporal y física entre los sujetos y en donde el cuerpo es concebido como un residuo instrumental de la actividad racional.

juveniles y géneros musicales (electro, minimal, tehcno, house, drum \& base, trace, entre otros), que marcan una búsqueda de experimentación e hibridación sonora constante. 
En contraposición, la cultura alternativa ${ }^{9}$, en lo que respecta al sentido del consumo de alcohol, comprende a este último como una sustancia de relajo, como una tecnología simbólica que opera flexibilizando la normatividad social que encubre el cuerpo durante el día y que lo distancia del contacto físico con otras/os. Y aquí se da un fenómeno relevante, en tanto el cuerpo vuelve a palparse, a ser visible a la experiencia subjetivas de las jóvenes, pero —a diferencia de la cultura electrónica/house — a su vez también se difumina, se disuelve en la trama general del carrete y del encuentro colectivo.

En línea con lo anterior, el cuerpo de las jóvenes de la cultura alternativa pasa a formar parte de una trama colectiva, dejando en segundo plano su dimensión individual y resintonizando con los ritmos grupales, en un proceso se encuentra entre el borrarse y el volver a identificarse en torno a una comunidad emocional, donde una se pone a tono con el resto generando lazos de confianza e intimidad. En ese sentido, el alcohol funciona como símbolo de apertura social en tanto inaugura la posibilidad de nuevas interacciones sociales que en un estado de sobriedad no se darían. Así, el alcohol adquiere el significado de lubricante social tal como en la cultura punk/hardcore — que no solo ayuda a forjar y reforzar lazos de intimidad y confianza dentro del grupo, sino que también funciona como mecanismo de inclusión que integra a otros y otras jóvenes al grupo, poniendo en paréntesis las dinámicas de sospecha y distancia que imperan en la vida cotidiana.

Así lo relata C.:

Y me pasa que cuando estái sobria no voy a andar por el mundo sociabilizando. Entonces, el copete te da la posibilidad de entablar relaciones con seres extraños (...). Soy más tela (...) No me siento tan ensimismada, tan encerrada. El copete me hace abrirme. (C., 27 años. Estudiante de Diseño Gráfico/vendedora. Puente Alto).

En consecuencia, el alcohol restituye la dimensión colectiva de los cuerpos y mediatiza las interacciones sociales, en un proceso que restituye el lazo social con el otro. Además, encontramos que el alcohol, al interior de esta cultura de consumo, desencadena un proceso de transgresión social y subjetiva, que abre espacios hacia otras sensaciones y experiencias:

Si quieres darlo todo, como mis últimos dos carretes de la vida, sí tenís que copetiarte porque lo pasái mejor. El copete vendría siendo un ente de alocamiento, perder el control y estar arriba de la pelota. (C., 25 años. Estudiante de Diseño Gráfico/vendedora. Puente Alto).

\footnotetext{
${ }^{9}$ El estilo alternativo, por tanto, se basa en la apropiación y reelaboración estética de la música de rock que, recogiendo estilos musicales de fines de los 70 y los 80 , como el punk, la new wave y el tecno, a comienzos de los 90 no era difundida en forma masiva por el circuito de la radio y la televisión chilenas. En ese sentido, la cultura alternativa está estrechamente vinculada a una performance estética que juega, a grandes trazos, en torno a una lógica de diferenciación con otros grupos a través de una identidad dramatizada en la ambigüedad sexual, la música de los 80 y la importancia del vestuario barato, pero cool. En tal sentido, las culturas de consumo alternativas aglutinan a grupos juveniles de estilos postpunk, new wave, skinhead, etc., que se congregan en torno a la música y al juego simulado de la exploración y la ambigüedad (Madrid, 2002). Dentro del perímetro metropolitano frecuentan discoteques como la Blondie, conocido punto del mundo alternativo, y algunos pubs del circuito central de diversión juvenil de la metrópoli, como son los barrios Bellavista y Yungay.
} 
El darlo todo, como expresión que metaforiza el consumo festivo que agota todo el capital económico y moral, a través del baile y del carrete. O también el estar on fire (prendido) comprende una puesta en escena, una dramatización del exceso, una exposición de un deseo desbordado que exige intensidad y la sublimación de la transgresión colectiva a través de besar o agarrase a alguien, o a más de una persona (pudiendo ser del mismo o distinto sexo); o bailar sin parar, sola o acompañada; o gastar todo el dinero que se trae para el carrete, tener sexo casual o solo conversar.

En consecuencia, la potencia de la desinhibición se amplifica en situaciones de borrachera, en donde las dinámicas de confianza e intimidad entre las participantes se refuerzan, generando cohesión e inclusión a un grupo identificatorio. A su vez, este proceso propicia la expansión y reapropiación de los espacios públicos y espacios intersticiales como territorios de desborde, como pliegues dentro de una geografía donde se ensayan y se practican otras lógicas de acción.

En el caso del efecto corporal y el significado social que se desprende del consumo de cocaína en estas culturas, este se basa en un uso similar, pero con ciertos matices, al que tiene la sustancia en la cultura electrónica/house. Dentro de este tipo de prácticas, la cocaína actúa como tecnología simbólica de energización complementaria. En otras palabras, la cocaína es ocupada en general al final de las cadenas rituales de consumo y sobre todo después de emborracharse, como una práctica para revivir, es decir, para contrarrestar el efecto físico depresor que tienen el alcohol y el policonsumo (sobre todo combinaciones de marihuana de tipo sativa con alto THC + destilados) en el cuerpo, propio de los carretes en discoteques, fuera de casas particulares. En consecuencia, la cocaína significa una sustancia que permite la continuidad de la transgresión (desinhibición, relajo, iniciativa) cuando ya se está en llegando a la última fase del carrete, y cuando el capital económico y la energía están terminándose para la joven y el grupo en general. De esa forma, el jalar funciona de manera ciertamente instrumental en las instancias de baile e interacción, en donde las jóvenes la consumen, ya sea en la pista de baile o en los baños para no morir, no dejar que el cuerpo vuelva a su estado de restitución biológica que supondría el desmayo por exceso de consumo de alcohol o el sueño como proceso de descanso. Precisamente, la cocaína, como símbolo de energía, es paulatinamente usada por las jóvenes que empiezan a trabajar y dejan la vida universitaria, en tanto las jornadas de trabajo son más extenuantes, lo que implica que al llegar a los carretes, ya sea en casa o en discoteque, hay menor energía y, por tanto, el uso de esta sustancia es más habitual.

\section{c) Espacios privados: intensificación oral y fuga}

En lo que respecta a los territorios privados, tales como cocinas, baños privados, etc., estos se construyen en torno a la intimidad y a vínculos de confianza o afecto entre los habitantes de ese territorio psicotrópico. En tal sentido, es en estos espacios donde se dan las condiciones de posibilidad para transgresiones sociales y morales libres de sanción, en el marco de un juego ritual a través del cual se pueden ensayar prácticas e interacciones sociales enmarcadas en guiones alternativos e incluso contrahegemónicos. En el caso de las culturas de estratos bajos, ya sean grupos de estilo underground ${ }^{10}$ o de grupos de amigas/os que se juntan en los hogares

\footnotetext{
${ }^{10}$ Los grupos de estilo underground nacen como forma de denominar a una heterogénea y amplia serie de grupos juveniles suburbanos que se encuentran fuera de la órbita mainstream (corrientes hegemónicas en términos de
} 
y que construyen circuitos de consumo periféricos asociados a la cumbia, el reggaetón o el vacile, el consumo de alcohol tiene un significado similar a las culturas anteriores, pero posee otro contexto.

En cuanto al efecto del alcohol, las jóvenes de estos estilos señalan que buscan la borrachera como objetivo del consumo, quedar happy (feliz) o prendías. Aquellos efectos corporales y subjetivos comprometen al igual que las culturas alternativas el vacilar, como esa práctica de socialización íntima y dinámica que se da entre amigos/as y que comprende tirar tallas (hacer bromas), reírse, conversar, gritar, bailar si se quiere o seducir y/o conquistar a algún hombre, según el marcado carácter heternormativo de este tipo de culturas de consumo.

Estas expresiones, en ese sentido, metaforizan una potencia expresiva del alcohol que se torna performance pública, desborde visible. Estas culturas tienen un acercamiento a los efectos del alcohol como parte de una experiencia de potencia que no se ensambla necesariamente con una determinada música, sino que se canaliza en torno a conversaciones, risas y bromas entre ellas o con otras/os. Tomar copete y curarse, por tanto, se comprende simbólicamente como prácticas de intensificación oral, a partir de la cual el diálogo y la palabra en sus distintas expresiones se transforman en la imagen del desborde. Simultáneamente, y de forma complementaria a la intensificación oral, el efecto del alcohol se traduce en prenderse, en tanto comprende una activación corporal que excede la normatividad y que se somete a una expresividad desenfrenada que desborda los códigos sociales de rigidez corporal, y en donde el cantar una canción favorita o gritar actúan como catarsis emocionales para las jóvenes. La risa y la ironía constituyen, en este estilo de culturas de consumo, elementos centrales en tanto actúan desacralizando jerarquías, develando contradicciones y visibilizando tabús sociales instituidos. A partir del humor, por tanto, es posible hablar de sexo, de relaciones de poder, de problemas cotidianos, como forma de hacerlos objeto de diálogo colectivo y darles un nuevo sentido como salida lúdica.

En lo que se refiere a los efectos de la cocaína, estos se codifican al igual que las otras como energía, pero también pa' despavilar:

En cambio, con el jale quedái despierto po’. Tai via, no te curái. (R., 21 años. Estudiante de Ingeniería Química. La Reina).

Estar vía representa un estado y sensación de alerta ante cualquier peligro o sanción. Así, estar vía comprende no tener una actitud pasiva ante los demás, sino que tomar un rol

estilo y visibilidad pública) y que se relacionan con industrias culturales que en un comienzo se consideraban propios de sectores populares (tatuaje, los piercings y estilos musicales como el rap, hip-hop, etc.). En Chile, la denominación underground se refiere más bien a una estética basada en la diferenciación de la estética mainstream, erigiendo modelos estéticos recogidos a través de páginas de internet o redes sociales, y que combinan el uso de piercings en las mujeres y tatuajes como símbolo de transgresión juvenil normalizada. Además, caracteriza a grupos de jóvenes de zonas periféricas y populares de la metrópoli que cultivan el arte del rap, la cumbia, grafiti o asociados a pequeños emprendimientos comerciales de comida, o diseño gráfico, que elaboran sus productos de manera casera, artesanal y con una reducida producción y, por tanto, circulan en circuitos de gente conocida o en ámbitos semipúblicos. En ese sentido, el underground se refiere más a una forma de hacer, una forma de mostrarse y una forma de construir un estilo de vida al margen de los circuitos de comercialización y ocio conocidos. 
activo de las situaciones, lo cual comprende saber manejarse, entender los códigos de interacción de la periferia y saber moverse en ellos.

En lo que respecta a la dimensión social de los efectos de la cocaína, las culturas de consumo periféricas ligadas a la cumbia, reggaetón y reggae tienden a tener una interacción social profunda, en la cual el baile se torna una experiencia colectiva de transgresión en la cual se dan coqueteos, miradas de unas a otras, de unas a otros, en donde se siguen coreografías aprendidas a través de la TV en la niñez, etc. La energía de la cocaína aporta la energía final para la consolidación de las cadenas rituales de consumo que habían empezado con marihuana y habían proseguido con cocaína. Sobre todo, los grupos exclusivamente de mujeres, construyen territorios psicotrópicos móviles y muy perceptibles a cualquier agente de control o disciplinamiento moral y social, por lo que han desarrollado estrategias de defensa. Aquí, el apañe, entendido como un compromiso irrestricto entre el grupo de jóvenes mujeres, se hace crucial para enfrentar posibles casos de violencia, detención policial o cualquier otro incidente. Por tanto, el jale aquí aparece como tecnología simbólica de energización asociada a la continuidad del baile como el reggaetón o la cumbia, pero también como estrategia de autodefensa para despertar y poder seguir carreteando o hacer frente a la situación de devolverse a la casa o encontrar una cama segura donde dormir. La transgresión del triple estigma moral que encarna el modelo señorita libre de drogas, no obstante, no comprende la superación de los contextos de violencia sexual y prácticas sexistas que ejercen estas culturas. En ese sentido, el jale tiene un uso recreativo e instrumental que apunta a volver a un estado de sobriedad que permita delimitar un límite de consumo y tomar decisiones que impidan episodios de violencia en contextos de transgresión festiva.

\section{CONCLUSiOnes}

Las culturas de consumo de la Región Metropolitana, tal como lo hemos señalado, son una serie de prácticas o modos de hacer en torno al consumo de sustancias psicotrópicas. Estas, tal como lo sostenemos en torno al proyecto, se entienden como tácticas de existencia que se basan en la gestión y alteración de la temporalidad y la corporalidad, como fundamentos de su accionar. En ese sentido, las distintas culturas de consumo operan en un contexto en el cual los espacios de la metrópoli están diseñados de antemano, por lo que han desarrollado principios que motivan una apropiación de esos espacios de poder para darles nuevos usos o solo para ocuparlos como práctica social de visibilización. Entre ellos, encontramos los principios de portabilidad, movilidad y contingencia como premisas básicas del consumo juvenil y de las instancias festivas.

En lo que respecta a las prácticas de consumo mismo, podemos afirmar que estas se estructuran en torno a cadenas rituales de consumo que articulan una experiencia y energía común, a partir de la cual se da la apropiación de espacios urbanos públicos, intersticiales y/o privados y la tensión de los códigos de uso de aquellos espacios. En término simbólicos, es posible leer los rituales de consumo como procesos dialécticos en que se da una dinámica centrípeta de autoafirmación de identidades individuales, grupales y/o generacionales ancladas en la comensalidad, como fundamento simbólico para la construcción de comunidades emocionales, a su vez que se da una dinámica centrífuga de exclusión y diferenciación simbólica basada en distinciones sociales. Precisamente en este punto, es posible visibilizar la acción del mercado como uno de los agentes contemporáneos más potentes en la constitución de las identidades juveniles y en la gestión de la población juvenil. 
Sumado a lo anterior, la dimensión social y moral del consumo nos da pistas de otros dispositivos de control que operan simultáneamente a los del mercado. Como revisamos, es posible sostener la existencia de un guion hegemónico que se basa en una normatividad social de género, que establece valores específicos para cada sexo en las distintas culturas de consumo. Así, vemos que este guion hegemónico produce y promueve, a través de distintas técnicas de normalización, un modelo de mujer en el campo de las drogas afín a los requerimientos del régimen neoliberal y que se traduce en el estereotipo de la señorita como canon moral de los procesos de subjetivación femenina, y en donde los mandatos de la abstinencia, la maternidad, la belleza, la suavidad, el recato y la heteronormatividad son elementos centrales para su constitución.

No obstante, y frente al despliegue de aquel guion hegemónico, encontramos prácticas y tácticas de transgresión, negociación, resistencia o rechazo a la normalización y a los dispositivos biopolíticos en curso. En este punto, y como parte de una multiplicidad de prácticas, encontramos la revitalización del cuerpo como soporte simbólico que impugna en un doble movimiento la matriz adultocéntrica de los distintos dispositivos de control y vigilancia social, y a su vez, la matriz patriarcal que existe al interior de sus grupos. El baile, los movimientos, las coreografías, etc., funcionan como forma de suspender la ley diurna de la sociedad.

En lo que respecta al propio consumo, entendemos las prácticas de las culturas de consumo como transgresiones rituales, una suerte de carnaval acotado y construido en los intersticios del tiempo de la rutina y que se constituye como un ejercicio prefigurativo, como distopía de vuelo raso que se circunscribe a una experiencia proteica, siempre mutable, en renovación y sucesión, que no termina de cristalizar, que siempre es inconclusa, que siempre deviene y que siempre se torna efímera pero intensa. Es, en cierta medida, la parodia de la rutina que intenta ser su espejismo transgredido, pero que en realidad funciona en línea de continuidad con aquella normalidad. En ese sentido, la utopía que se da en el carrete es siempre paradojal: es la prefiguración de una transgresión intraducible, de una fuga que no termina de nunca ser exitosa, pero que en ese ensayo recrea nuevas formas de existencia.

Finalmente, y en relación con la transgresión femenina en sí misma y su estatuto dentro de las culturas de consumo metropolitanas, es necesario resituar estos quiebres en los procesos de (des)masculinización de ciertos espacios y cómo la integración de mujeres u otros sujetos marginados despliega procesos de conflictividad simbólica y social. En esa dirección, es posible afirmar que las prácticas de consumo de las jóvenes operan como una transgresión dentro de la misma transgresión que representan los espacios festivos como espacios de creación y exploración de narrativas desviadas del guion hegemónico. No obstante, la transgresión del campo de las drogas como territorio masculinizado gatilla también resistencias de parte de los hombres, las que se traducen en episodios múltiples de violencia que tienden a reasegurar las relaciones de poder en torno a las culturas de consumo mixtas o con otros jóvenes, en un hecho que visibiliza el carrete como un locus clave en los procesos de construcción de género de las juventudes metropolitanas. En ese sentido, cabe continuar desnaturalizando los espacios de ocio y diversión como espacios exentos de violencia y rebosantes de libertad, y en donde se (re)producen relaciones de poder, jerarquías ancladas a desigualdades y diferencias notorias en torno a los sexos, los posicionamientos socioeconómicos, generacionales y territoriales.

RECIBIDO: 30 DE MARZO DE 2018 
ACEPTADO: 9 DE DICIEMBRE DE 2018

\section{REFERENCIAS BIBLIOGRÁFICAS}

AlpízAR, L. y Bernal, M. (2003). La construcción social de las juventudes. Última Década, 11(19), 105-123.

Aguilera Ruiz, Ó. (2014). La idea de juventud en Chile en el siglo XX. Aproximación genealógica al discurso de las revistas de juventud. Anagramas Rumbos y Sentidos de la Comunicación, 12(24), 141-159.

BABCOCK, M. (1996). Does feminism drive women to drink? Conflicting themes. International Journal on Drug Policy, 7, 158-165.

BIERNACKI, P. y WALDORF, D. (1981). Snowball sampling: Problems and techniques of chain referral sampling. Sociological methods \& research, 10(2), 141-163.

Collins, R. e Iranzo, J. M. (2009). Cadenas de rituales de interacción. Barcelona: Anthropos.

Comisión Interamericana para el Control del Abuso de Drogas (CiCAD) (2015). Hemispheric Report. Evaluation Report on Drug Control. Washington D.C.: Organization of American States.

Del Zotto, P., Cortés, M. T., Gómez, R. y Luque, L. (2010). La realidad intercultural del consumo intensivo de alcohol en jóvenes españoles y argentinos. VI Congreso Iberoamericano de Psicología. Oviedo, España.

DUARTE, K. (2000). ¿ Juventud o juventudes? Acerca de cómo mirar y remirar a las juventudes de nuestro continente. Última Década, (13), 3.

ETTORE, E. (1992). Women and Substance Use. Houndsmills: Macmillan.

FEIXA, C. (2006). Generación XX. Teorías sobre la juventud en la era contemporánea. Revista Latinoamericana de Ciencias Sociales, Niñez y Juventud, 4(2), 21-45.

FERnÁNDEZ LABBÉ, M. (2011). Drogas en Chile. 1900-1970: Mercado, consumo y representación. Santiago: Universidad Alberto Hurtado.

FouCAULT, M. (1990). Las tecnologías del yo y otros textos afines. Barcelona: Ediciones Paidós Ibérica.

Funes, J. y Romaní, O. (1985). Dejar la heroína. Vivencias, contenidos y circunstancias de los procesos de recuperación. Madrid: Cruz Roja Española.

García-Mina, A. y CarRasco, J. (2007). Diferencias de género en el consumo de drogas. Madrid: Editorial Universidad Pontificia Comillas. 
GHiARDO, F. (2003). Acercándonos al sentido del uso de drogas y la prevención desde los jóvenes. Última Década, 11(18), 123-151.

GómEZ, R. (2012). Drogas y drogodependencias, elementos para una perspectiva histórica compleja. En Introducción al campo de las drogodependencias. Córdoba: Editorial Brujas.

Le Breton, D. (1990). Antropología del cuerpo y modernidad. Buenos Aires: Nueva Visión.

LUNA-FABRitius, A. (2015). Modernidad y drogas desde una perspectiva histórica. Revista mexicana de ciencias políticas y sociales, 60(225), 21-44.

MAtus, CH. (2005). El carrete como escenario: una aproximación etnográfica a los códigos de la sexualidad ocasional en jóvenes urbanos. Última Década, 13(22), 09-37.

MArcus, G. (2001). Etnografía en/del sistema mundo. El surgimiento de la etnografía multilocal. Alteridades, 11(22), 111-127.

Markez, I. y G. Silvosa Rodríguez, G. (2011). Drogas y género en el ámbito penitenciario. Oñati Socio-Legal Series, 2(6), 146-160.

McDonald, M. (1994). Introduction. A Social-Anthropologicla View of Gender, Culture and Drugs. En M. McDonald (Ed.), Gender, Drink and Drugs. Cross Cultural Perspectives on Women. Oxford: Berg Publishers.

MuÑIZ, E. (2014). Pensar el cuerpo de las mujeres: Cuerpo, belleza y feminidad. Una necesaria mirada feminista. Sociedade e Estado, 29(2), 415-432.

RALET, O. y Grupo IgIA (2000). Condicionantes políticos y económicos: Análisis de la influencia de estos factores en la construcción social del problema de la droga. Contextos, Sujetos y Drogas. Un manual sobre Drogodependencias, 43-51.

RoMANí, O. (1999). Las drogas, sueños y razones. Barcelona: Ariel.

Romo, N. y PÉRez, N. (2013). ¿Las chicas también arriesgan? En De riesgos y placeres. Manual para entender las drogas. Lleida: Milenio.

Rosenbaum, M. y MuRPHY, S. (1990). Women and addiction: process, treatment and outcome. En E. Y. LAMBERT (Ed.), The collection and interpretation of data from hidden populations. Rockville: US Department of Health and Human Services.

Rubin, G. (1986). El tráfico de mujeres: Notas sobre la «economía política» del sexo. Nueva Antropología, 8(30), 95-145.

Servicio Nacional para la PreVención y Rehabilitación del Consumo de Drogas y AlCohol (SENDA) (2016). Décimo Primer Estudio Nacional de Drogas en Población General de Chile, Santiago. 
(2015). Décimo Estudio Nacional de Drogas en Población General de Chile, Santiago.

(2012). Noveno Estudio Nacional de Drogas en Población General de Chile. Santiago.

SpotTs, J. V. y Shontz, F. C. (1980). A life-theme theory of chronic drug abuse. NIDA research monograph, 30, 59-70.

SPREEN, M. (1992). Rare populations, hidden populations, and link-tracing designs: What and why? Bulletin of Sociological Methodology/Bulletin de Methodologie Sociologique, 36(1), 3458.

Tolvanen, E. y JYlHä, M. (2005). Alcohol in life story interviews with Finnish people aged 90 or over: Stories of gendered morality. Journal of Aging Studies, 19(4), 419-435.

Valenzuela, J. E. B., De Mello, C. M. y Vieira, F. G. D. (2009). Consumidores: una reflexión sobre los aspectos de la cultura del consumo. Revista de Administração da UnimepUnimep Business Journal-B2, 7(1), 105-121.

Vigarello, G. (2005). Historia de la belleza. El cuerpo y el arte de embellecer desde el Renacimiento hasta nuestros días. Buenos Aires: Nueva Visión.

Wilsnack, R. W., Vogeltanz, N. D., Wilsnack, S. C. y HarRis, T. R. (2000). Gender differences in alcohol consumption and adverse drinking consequences: Crosscultural patterns. Addiction, 95, 251-265. 